\title{
The Cognitive Processes used in Team Collaboration during Asynchronous, Distributed Decision Making
}

\author{
Norman W. Warner \\ Naval Air Systems Command \\ Human Systems Department \\ Code 4.6.5.3 \\ Building 2187, Suite 2259 \\ Patuxent River, Maryland 20670 \\ Office: (301) 342-9288 \\ Fax: (301) 342-9305 \\ norman.warner@,navy.mil
}

\author{
Elizabeth M. Wroblewski \\ Naval Air Systems Command \\ Human Systems Department \\ Code 4.6.5.4, \\ Building 2187, 2280-C6 \\ Patuxent River, Maryland 20670 \\ Office: (301) 342-9286 \\ Fax: (301) 342-9305 \\ elizabeth.wroblewski@,navy.mil
}




\begin{abstract}
The purpose of this paper is to describe the unique cognitive processes that are employed to optimize collaborative team decision making in a geographically distributed and time delayed situation. The cognitive processes will be illustrated within the context of a structural model of team collaboration. The team collaboration model has four unique but interdependent stages of team collaboration. The stages are: Team Knowledge Construction, Collaborative Team Problem Solving, Team Consensus, and Product Evaluation \& Revision. The stages are not strictly sequential but are very dynamic throughout the collaboration process. The cognitive processes are represented at four levels: Meta-cognition, which guides the overall problem solving process, the Information Processing Tasks required by the team to complete each collaboration stage, the Knowledge Required to support the information processing tasks and the Communication Mechanisms for Knowledge Building and Information Processing.

Results from the empirical team collaboration studies indicate significant differences in the cognitive processes and sub-processes across the four collaboration stages for the face-to-face teams compared to asynchronous, distributed teams. Understanding these differences will facilitate the design of collaboration tools for asynchronous, distributed teams and will provide for more effective and timely collaborative decision-making.
\end{abstract}

\title{
Introduction
}

The structural model of team collaboration described in this paper emphasizes the cognitive aspects of the collaboration process. The model includes the domain characteristics, collaboration stages, meta-cognitive processes, information processing tasks, knowledge required for each information task and the communication mechanisms for knowledge building and information tasks. There have been numerous models of team collaboration (Orasanu \& Salas, 1992; Rogers \& Ellis, 1994; Stahl, 2000; McNeese, Rentsch, Perusich, 2000; Hurley, 2002; Noble, 2002) each focusing on various aspects of collaboration while describing those aspects at different levels of detail. However, for a model of collaboration to be an effective mechanism for understanding the operating cognitive mechanisms underlying collaborative team behavior the model needs to be defined at a level of granularity, which covers all the major components and mechanisms of team collaboration. The approach to describing the current model is as follows: (1) define the problem domain for the model, (2) define all the various collaboration stages that a team goes through to solve the problem, (3) define the metacognitive processes that guide team collaboration, (4) define and describe the information processing components that the team performs to achieve each collaboration stage, (5) define the knowledge required to achieve each information processing component and (6) define the communication mechanisms used by the team to build the necessary knowledge along with supporting the information processing. The cognitive mechanisms in the model are described at a macro level (i.e. meta-cognition,information processing, knowledge building and communication mechanisms) rather than at the micro level (i.e. neural-cognitive). The reason for describing the model's cognitive processes at the macro level is driven by our limited understanding of how teams think during collaborative problem solving in asynchronous, distributed environments. In addition, our current metrics for measuring, at the micro level, the cognitive mechanisms teams use to solve collaborative problems are still pre-mature and need further refinement. The model's 
macro level definition of the cognitive processes permits empirical assessment of these cognitive processes using our current measurement techniques (e.g. verbal protocol, communication analysis).

Before getting into a detailed model description it is important to understand the scope of the problem area that the collaborative model will be addressing. Figure 1 illustrates the major factors impacting military collaborative teams. These factors include the Collaborative Problem Environment, Operational Tasks, Collaborative Situation Parameters, and Team Types. The collaborative problem environment has grown in complexity over the past decade (Jensen, 2002). The military problems are becoming more complex requiring teams to address the problems. In addition, problems are addressed at an international level requiring agile coalition operations. Developments in information and communication technologies have provided greater communication between coalitions but information overload is still a problem due to a lack of information management (Information Management Strategic Plan, 1999). There are many operational tasks, which involve team collaboration (Jensen, 2002; Joint Vision 2010, 2002). However, to gain an understanding of the team collaboration process the model will focus on two operational tasks: (1) team decision-making, course of action selection, and (2) Intelligence analysis (team data processing). During team collaboration there are various parameters that can influence collaboration performance (Warner, Letsky \& Cowen, 2003). However, the collaborative situation parameters listed in Figure 1 were chosen as the critical parameters to focus our collaboration domain because of their significance to current military requirements (Jensen, 2002). The final factor is team types, which of all the factors has the most number of different categories that can influence collaboration performance. The 7 team type characteristics listed in Figure 1 were selected based on the common characteristics of today's military collaborative teams (Jensen, 2002). In summary, the problem area domain for our structural model of team collaboration can be defined by the respective characteristics under operational tasks, collaborative situation parameters, and team types specified in Figure1.

In addition to understanding the model's problem domain, it is also important to understand how current theories of cognitive psychology impact the model definition and design. One of the key issues in developing a collaboration model is to understand the cognitive processes that team member's use as they perform the various stages of collaboration. To understand these cognitive processes it is necessary to examine the various theories of cognitive psychology. Early philosophers such as Descartes (1641) and Kant (1781) have examined basic questions of the origin of knowledge and human thought. However, it was not until after the failure of behaviorism (Skinner, 1985,1989) to explain internal representations that cognitive psychology emerged. A multitude of theories of human cognition began to develop. Several theories (Turing, 1936; Weiner, 1948; Shannon, 1949; Wickens, 1992) explain human cognition in terms of an information-processing model, which focus on information representation, processing and computation. Other theories (Chomsky, 1957; Cooke, 2003) believe that the development of knowledge and the processes of thought are achieved through human language. Piaget's (1970b) focused on developmental biology, which described not only the different components of human cognition but also the developmental stages of 


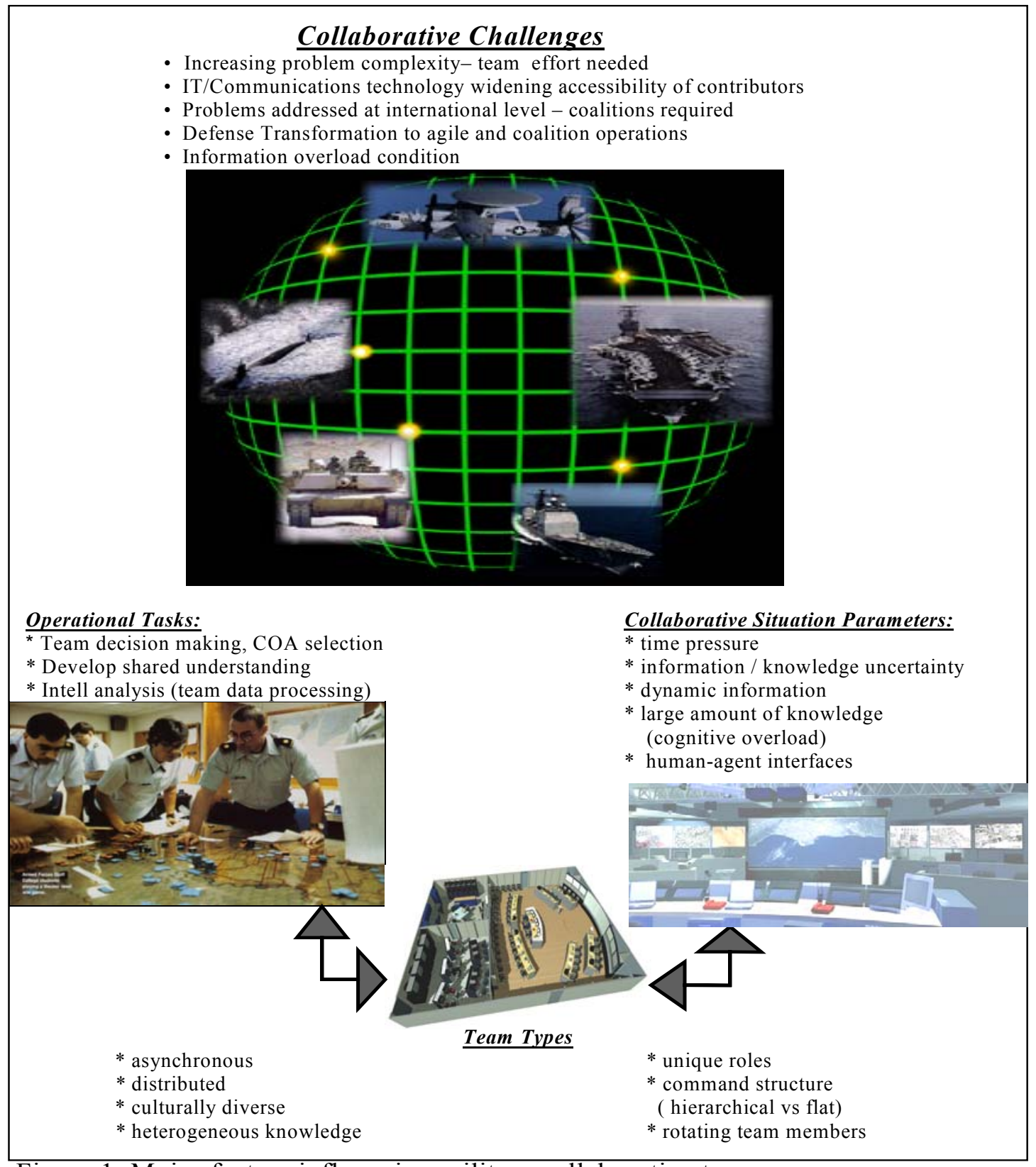

Figure 1: Major factors influencing military collaborative teams

cognition. As computer science matured, several theories developed (Newell \& Simon, 1956; Feigenbaum \& Feldman, 1963; Anderson, 1993; Minsky, 1997) that explain human cognition in terms of a computer computational model. These computational models varied in how they explained cognition ranging from computational logic, production rules (e.g. If X Then Y), to frames (data structures). Other theories (Rumelhart, 1990; Churchland, 1989; Rosenburg, 1988) emphasize physiology in understanding human cognition. These theories use physiological neural networks represented in computational models to explain cognition and its processes. Davidson, Deuser \& Sternberg (1994) proposed a theory of meta-cognition, which is knowledge of one's own cognitive processes, in explaining how human cognitive processes are used in problem solving. Each theory has provided unique insight and empirical data to explain various aspects of human cognition and its processes. Current research in human cognition uses a multidisciplinary approach by considering ideas from all the relevant disciplines including philosophy, psychology, linguistics, computer science, anthropology, cognitive 
neuroscience, and artificial intelligence. However, even with this multidisciplinary approach there is no generally recognized unified theory of human cognition. This lack of a unified theory is partially due to the recent integration of the various disciplines in studying human cognition along with insufficient objective metrics to measure the cognitive processes. The challenge in representing the cognitive components in a collaboration model is deciding what theoretical approach(s) to utilize during initial model development. There is insufficient empirical research describing the actual cognitive processes that teams use during collaborative problem solving especially under conditions specified in Figure 1. As a result of the current state of the various theories of human cognition, the cognitive components of the team collaboration model shall be described using current theories of meta-cognition, human information-processing and human communication. These theoretical approaches were selected based on our current ability to measure how teams use communication (i.e. written and verbal) to process information and build knowledge during collaborative problem solving. This level of granularity in describing the cognitive processes in team collaboration permits empirical evaluation of the various components of the model. However, this level of granularity will not provide a comprehensive view (e.g. neural components) of the cognitive processes used in team collaboration. A detail description of the model's cognitive components and processes are presented under the model section of the paper.

In describing the team collaboration model it is important to understand what the term model means along with understanding the objectives of the model. A model is not the system or process itself but an abstract representation that enables researchers to predict behavior and to test hypotheses. Models may be mathematical, physical, or structural. For example, information theory and signal detection theory are mathematical models; neuroanatomical models are examples of physical models, while information-processing models are examples of structural models. Due to the lack of sufficient objective metrics for measuring cognition, most models in cognitive science are either structural or mathematical not physical models. The objectives of the proposed team collaboration model are: (1) to understand the cognitive mechanisms and their relationships during team collaborative problem solving, (2) to provide a model-based approach to experimentation of team collaboration, (3) to identify and prioritize required areas of research in team collaboration, and (4) to serve as a design guideline for an agent-based support tool for team collaboration.

\section{Structural Model of Team Collaboration}

Figure 2 presents the structural model of team collaboration. The model's domain is defined by the problem area characteristics, which were described earlier in this paper. The model consists of general inputs (e.g. task description, team roles), collaboration stages that the team goes through during the problem-solving task, the cognitive processes used by the team and final team output(s) (e.g. selected course of action, recommendations). The four cognitive processes include: (1) the meta-cognitive processes, (2) the information processing tasks, and (3) the knowledge required to support the information tasks, and (4) the communication mechanisms for knowledge building and information processing. 


\section{STRUCTURAL MODEL OF TEAM COLLABORATION}

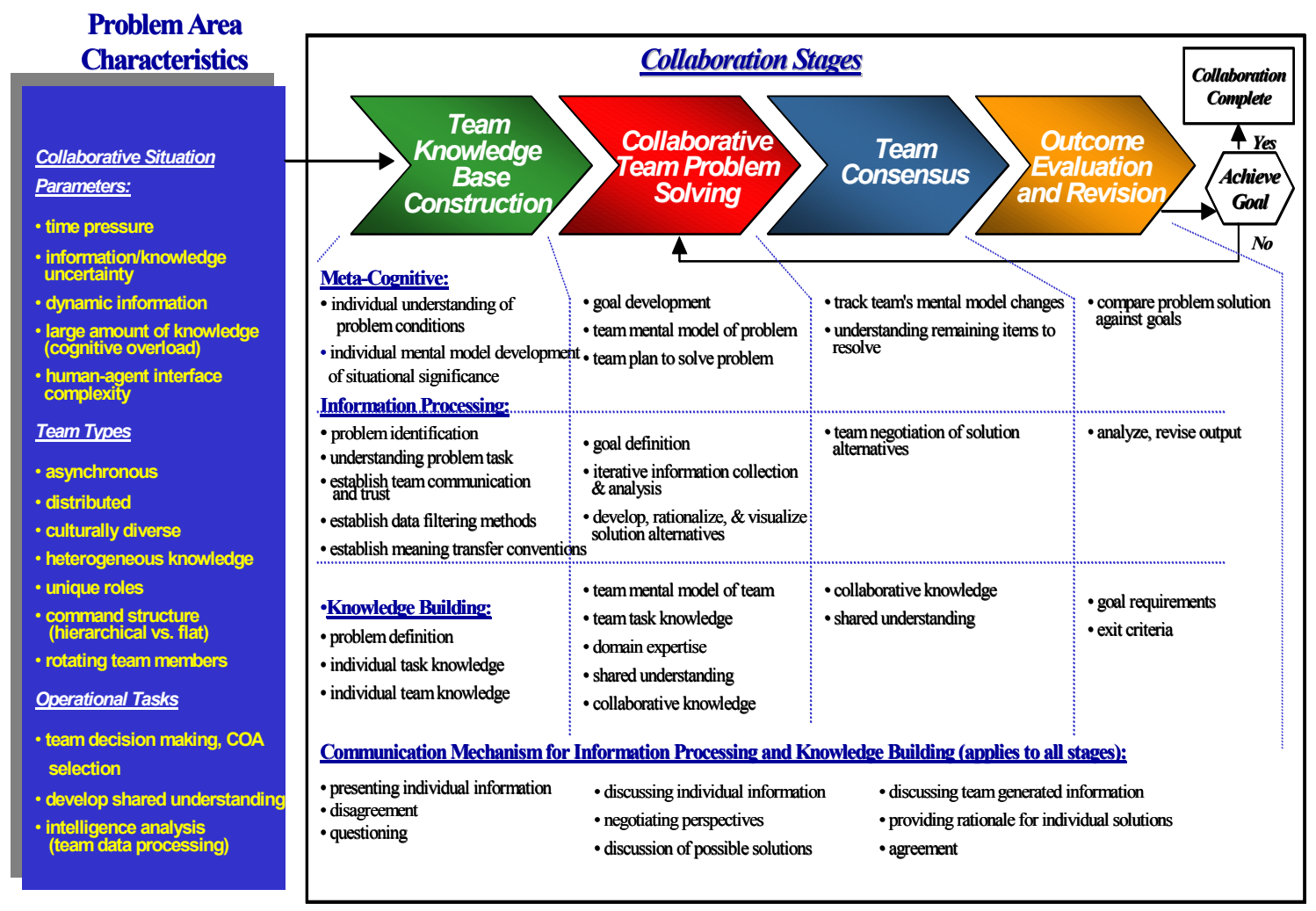

Figure 2: Structural Model of Team Collaboration.

The model is a synthesis of the literature in team collaboration, human information processing and team communication together with the results obtained during the annual workshop on Collaboration and Knowledge Management (Letsky, 2003). During the Collaboration and Knowledge Management (CKM) workshop twelve initial conceptual models of team collaboration were produced each providing some unique information along with overlapping information. The models varied in their approach from information-processing, team recognition primed decision making, transactive memory, discovery and innovation, and hybrids including multi-stage and process models. The collaboration stages within the model were selected by determining the minimum number of unique stages identified in the team collaboration literature together with the twelve models. These stages also had to be supported by some empirical research. The cognitive processes required during team collaboration are based on an information-processing approach, which also includes knowledge building for supporting the information processing tasks. In this model knowledge is built through team communication with the initial communication mechanisms derived from previous research (Stahl, 2000; Cooke, 2003; Warner, Vanderwalker and Verma, 2003).

\section{Model Components}

Inputs to Model. These inputs represent general information that is required prior to team collaboration. This information includes such items as: (1) a description of the problem task to be solved, (2) team member expertise, (3) organizational structure, (4) 
team members roles and responsibilities and (5) projected events/future information. This representative domain information is provided to the team during team formation.

Collaboration Stages and Cognitive Processes. The model has four unique but interdependent stages of team collaboration. The stages are: Team Knowledge Base Construction, Collaborative Team Problem Solving, Team Consensus and Product Evaluation \& Revision. There is also a feedback loop for revising team solutions. The stages are not strictly sequential as it may appear in Figure 2. The team will start in the Team Formation stage and proceed onto Collaborative Team Problem Solving, Team Consensus and Product Evaluation \& Revision. However, team communication is very dynamic and moves across the stages throughout the collaboration process. The cognitive processes are represented at four levels: Meta-cognition, which guides the overall problem solving process, the Information Processing Tasks required by the team to complete each collaboration stage, the Knowledge Required to support the information processing tasks and the Communication Mechanisms for Knowledge Building and Information Processing. For each collaboration stage the respective supporting cognitive processes will be described.

Team Knowledge Base Construction is the first step in team collaboration and begins by identifying the relevant domain information required, selecting the required team members and setting up the communication environment necessary to address the problem. The type of teams representative in this model have characteristics such as being asynchronous, distributed, culturally diverse, having heterogeneous knowledge, and rotating team members (see Problem Area Characteristics for complete list).

Meta-cognitive Process: The meta-cognitive component in Team Knowledge Base Construction is the Team's understanding of the elements, relations and conditions that compose the initial state of the problem. Also part of this meta-cognitive process is for each team member to construct an individual mental model for use in developing team understanding.

Information Processing Tasks: The team has several information processing tasks to perform during the team formation stage. The team has to collaborate to identify the problem to be solved, to understand the problem task, and establish team communication and team trust. In identifying and understanding the problem task the team must recognize that a problem exist before they can solve it. Critical elements of the problem need to be encoded in short-term memory while information relevant to these elements are retrieved from long-term memory. The team can then collaborate on what is known, unknown and what is being asked in the problem situation.

Knowledge Required: Three types of knowledge need to be built and used by the team members in support of completing the information tasks. Each team member needs to develop his or her individual knowledge of the task. The team, as a whole, needs to develop the team's knowledge of the task. Individual and team task knowledge does not have to be exactly the same. The third knowledge type is the team's knowledge of the problem to be solved. 
Communication Mechanisms for Knowledge Building and Information Processing: In order for the team to build the required knowledge needed to support the information processing tasks the team will use verbal and /or written communication between team members. The team when collaborating on the information processing tasks uses these same mechanisms. The specific communication mechanisms are: presenting individual information, discussing individual information, discussing team generated information, negotiating perspectives, providing rationale for individual solutions, agreement, disagreement, questioning and discussion of possible solutions.

Collaborative Team Problem Solving is the next stage and begins following completion of the Team Knowledge Base Construction information processing tasks. The collaborative Team Problem Solving stage is where the majority of collaboration occurs among team members. The team's main objective in this stage is to develop viable solutions to the problem.

Meta-Cognitive Process: The meta-cognitive processes involved in this stage includes, overall goal development, developing a team mental representation of the problem and planning how the team will solve the problem. The team mental representation can change during the course of solving the problem. Changes can occur as the team gains more complete understanding of the problem elements, goals or overlooked information. According to Davidson and Sternberg (1984, 1986), new mental representations are constructed through three related mental processes: selective encoding, selective combination, and selective comparison. Selective encoding restructures the team mental representation so that information that was originally viewed as being irrelevant is now seen as relevant for problem solution. Also, information that was originally seen as relevant may now be viewed as irrelevant and eliminated from the team's mental representation. Selective combination involves putting together elements of a problem task in a way that previously was not obvious to the team. This new way of combining the problem elements results in a change in the mental representation. Selective comparison involves discovering relationships that are not obvious between new information and information acquired in the past. The realization of a relationship between new and old information results in a change to the mental representation. After the problem has been identified and mentally represented, the team must decide which steps to use in solving the problem. Planning often involves dividing the problem into sub-problems and then devising a sequence for how the sub-problems should be completed (Flower \& Hayes, 1981). There are three characteristics of planning (Pea \& Hawkins, 1987). First, the team is more likely to engage in planning when the problem situation is novel and complex because there are no known strategies to follow. Second, planning tends to be abstract rather than concrete because the team revises their plan based on how well it is working and available opportunities for modifications. Third, plans have both costs and benefits, which involves time and cognitive resources but overall improve problem solving efficiency. In team problem solving there are four heuristics that are often used (Greeno \& Simon, 1988). One is the means-ends analysis, which tries to decrease the distance between the team's current position in the problem space and where the team wants to go in that space. A second heuristic is working 
forward, which involves starting at the initial problem state working toward the desired state. The third heuristic is working backward, which involves starting at the desired state and trying to work back to the initial state. The fourth heuristic is generate and test, which involves generating alternative courses of action and evaluating whether each course will work.

Information Processing Tasks: In order to develop problem solutions the team performs several information processing tasks. These tasks include definition of the team goal, iterative information collection and analysis, and development, rationalization and visualization of solution alternatives.

Knowledge Required: Five types of knowledge are required in support of completing the information tasks. Each team member needs to continue to develop his or her individual knowledge of the task. The team, as a whole, needs to continue to develop the team's knowledge of the task. The team in addition needs to develop shared understanding (i.e. joint agreement of the facts and recognition of team member perspectives but not joint acceptance). Collaborative knowledge (i.e. team negotiation of perspectives resulting in a deeper understanding and team agreement of the facts) also needs to be developed by the team. The last knowledge type is domain expertise, which is not developed by the team during collaboration but is required by team members to successfully perform the information processing tasks.

Communication Mechanisms for Knowledge Building and Information Processing: During the Collaborative Team Problem Solving stage the team will use some or all of the communications mechanisms discussed earlier to perform the information processing tasks and knowledge building.

Team Consensus is the next collaboration stage and begins when the team has several viable solution alternatives to the problem. The main objective of team consensus is to achieve team agreement of the common output.

Meta-cognitive Process: The meta-cognitive processes involved in this stage includes, the team keeping track of what they have already done, what they are currently doing and what needs to be done. In other words, the team needs to track changes to the team's mental model.

Information Processing Tasks: In order for the team to achieve agreement of the common output, the team's mental model of the solution must converge. The information-processing task under this stage is team negotiation of the solution alternatives.

Knowledge Required: To achieve convergence of the team's mental model and successful team negotiation of solution alternatives two types of knowledge are required: (1) shared understanding (i.e. joint agreement of the facts and recognition of team member perspectives but not joint acceptance) and (2) collaborative knowledge (i.e. team 
negotiation of perspectives resulting in a deeper understanding and team agreement of the facts).

Communication Mechanisms for Knowledge Building and Information Processing: During the Team Consensus stage the team will use some or all of the communications mechanisms discussed earlier to perform the information processing tasks and knowledge building.

Outcome Evaluation and Revision is the final stage of collaboration. The main objective of this stage is to analyze, test and validate the agreed upon team solution against the goal requirement(s) and exit criteria. Included in this stage is an iteration loop for deriving other solutions for the problem if necessary.

Meta-cognitive Process: The meta-cognitive processes involved in this stage include comparing the problem solution against the goal(s).

Information Processing Tasks: The information-processing task under this stage is for the team to analyze and revise the problem solution, if necessary.

Knowledge Required: In order for the team to perform the informationprocessing task, two types of knowledge are required: (1) goal requirements and (2) exit criteria for viable solutions.

Communication Mechanisms for Knowledge Building and Information Processing: During the Outcome Evaluation \& Revision stage the team will use some or all of the communications mechanisms discussed earlier to perform the information processing tasks and knowledge building.

Model Outputs. The output of the model reflects the type of product from the team collaboration process. The product type will vary depending on the problem domain addressed by the team. This structural model of team collaboration focuses on the following product types: selected course of action(s), recommendations, situation assessment, risk assessment, product or tool, opinion, guidelines.

The proposed structural model of team collaboration is intended to be a starting point for understanding the cognitive mechanisms of team collaboration given the collaborative problem area characteristics presented in Figure 2. This model also offers a model-based approach to experimentation of team collaboration, which aids in focusing specific research experiments while enhancing the model through new research findings. The following experiment was designed to provide empirical data on the validity of the collaboration stages and cognitive processes of the structural model. The experiment had two independent variables, Collaboration Mode (i.e. face-to-face and asynchronous, distributed) and Knowledge Distribution (i.e. Homogeneous and Heterogeneous). Results from the experiment were used to update the structural model of team collaboration. 


\section{Method}

\section{Participants}

Eighty-four undergraduate students from a community college in Southern Maryland served as participants in this study. Participants included twenty-three men and sixty-one women, ranging in age from 16 to 58 years of age (mean $=22.92$ years, standard deviation $=8.76$ ). Participants were paid $\$ 15$ per hour for a task that could last up to four hours and received extra credit from their professor. Students were randomly assigned either to the face-to-face, homogeneous teams ( $n=$ seven teams), face-to-face heterogeneous teams $(n=$ seven teams), asynchronous, distributed homogeneous teams $(\mathrm{n}=$ seven teams) or asynchronous, distributed heterogeneous teams ( $\mathrm{n}=\mathrm{seven}$ teams). Each team consisted of three students.

\section{Apparatus}

Both the face-to-face and asynchronous, distributed teams solved the collaborative problem solving task in the Naval Air Systems Command Cockpit Automation and Research Laboratory. Figure 3 and 4 illustrate the face-to-face and asynchronous, distributed environments respectively.

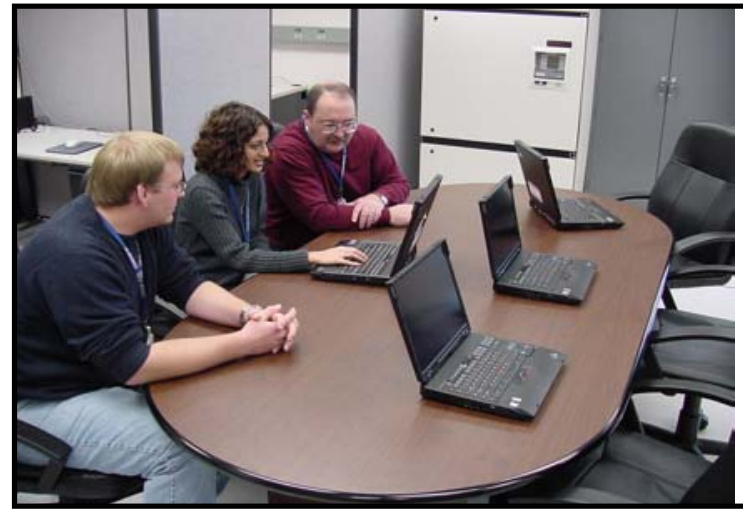

Figure 3: Face-to-Face Environment

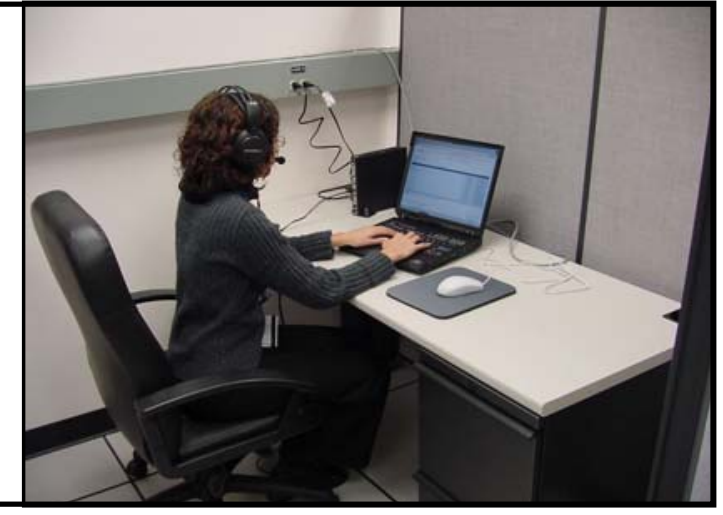

Figure 4: Asynchronous, Distributed Environment

\section{Experimental Task}

The experimental task required participants to work in teams of three to solve a murder mystery. After reading The Case of the Fallen Businessman (Stasser, 1995), the teams were required to reach consensus, identifying which of the three possible suspects they believed committed the murder. During collaborative problem solving the face-to-face teams solved the mystery through direct speech discussions around a conference table while the asynchronous, distributed teams used a web-based text forum to communicate with other team members. To simulate an asynchronous environment, a randomized delay between 1 and 60 seconds was used to delay the message from the time it was posted by a team member to the time it was displayed on the forum. Team members assigned to the homogenous groups received identical information about the murder mystery. Participants assigned to the heterogeneous groups received common information as well as information unique to each team member. Heterogeneous team members were not informed of the script variations. 
The web-based forum was hosted on a PC server and presented to asynchronous, distributed team members on their IBM laptop. All asynchronous, distributed team members were in separate office modules within the laboratory. All face-to-face communication was audio and video recorded and timed tagged for later transcription while all the web-based forum text was recorded, time tagged and saved real-time.

\section{Procedure}

After being assigned to one of the four experimental groups (face to face homogeneous, face to face heterogeneous, asynchronous distributed homogeneous or asynchronous distributed heterogeneous), participants reviewed and completed informed consent forms. All teams were then given a brief introduction to the murder mystery task. In addition, asynchronous distributed teams were instructed on the use of the web-based forum. Following the instruction period, they were outfitted with hearing protection, seated at an isolated computer station and given a five-minute practice session. Following the procedural introduction, team members were given twenty minutes to independently read The Case of the Fallen Businessman (Stasser, 1995). The script given to them was specific to their assigned knowledge distribution (homogeneous or heterogeneous). While subjects were allowed to take notes when reading, interaction and discussion among subjects was prohibited. Participants were then instructed to reach a group consensus regarding the identity of the murderer. Previous studies (Cristian,1996; Goodman,et.al.,2001) comparing face to face and asynchronous, distributed collaborative teams have shown that face to face teams complete collaborative problems faster than asynchronous, distributed teams. For this experiment this effect was to be minimized. Each of the four experimental groups had a maximum time to complete the problem. The maximum collaboration time allowed varied with each experimental group based on previous pilot studies. Face to face homogeneous teams were allowed 15 minutes to reach a consensus, while face to face heterogeneous groups were allowed 30 minutes. Likewise, asynchronous homogeneous groups were allowed 60 minutes for consensus and asynchronous heterogeneous groups were given 75 minutes. Groups that reached consensus before the allotted time expired were allowed to end the session.

\section{Dependent Variables}

Five dependent variables were identified:(1) accuracy of decision, (2) time for task completion, (3) time spent in each collaboration stage and cognitive process state, (4) frequency of utterances, and (5) transition probabilities.

Accuracy of Decision: The collaborative task was designed to provide all of the information necessary to correctly identify a specific suspect as the murderer. Performance was assessed through a comparison of the percentage of correct decisions by collaboration mode and knowledge distribution.

Time for Task Completion: In an effort to compensate for variations in task difficulty, the maximum collaboration time allowed varied with each experimental group. The adjusted mean percent of time for task completion was assessed in relation to collaboration mode and knowledge distribution. This was accomplished by comparing the time allotted to the actual time used for each team. 
Time Spent in each Collaboration Stage and Cognitive Process State: The time each team spent in each collaboration stage and process state was analyzed with respect to the total time needed for task completion.

Frequency of Utterances: An analysis of the number of utterances within each collaboration stage and process state was performed.

Transition Probabilities: Transition probabilities indicate the likelihood that one collaboration stage will follow another. Transition probabilities for cognitive process states were also analyzed.

\section{Results}

A Chi Square test of independence was used to analyze accuracy of team decisions across collaboration mode and knowledge distribution conditions. A 2x2 randomized factorial analysis of variance was used to analyze time for task completion across collaboration mode and knowledge distribution along with time spent and frequency of utterances in collaboration stages. Two trained raters using verbal protocol analysis with a pre-defined coding scheme to categorize each utterance with respect to collaboration stage and process state performed the initial analysis of the collaboration stages and cognitive processes. The specific coding categories and their definitions are described in appendix A. Multiple analysis of variance (MANOVA) analyses were used to analyze time spent along with frequency of utterances in each cognitive process state. For all statistical analyses an alpha level of .05 was used for statistical significance.

There was no significant effect of decision accuracy across collaboration mode and knowledge distribution conditions, Yates corrected Chi Square $(1)=.27, p=.6056$. There was no significant effect for mean percent time for task completion across collaboration mode, $\mathrm{F}(1,82)=0.6406, \mathrm{p}=0.431353$ or knowledge distribution, $\mathrm{F}(1,82)=0.1491, \mathrm{p}=$ 0.702782 , or the interaction, $\mathrm{F}(3,80)=0.0006, \mathrm{p}=0.933647$. Figure 5 illustrates a significant effect of the mean percent time spent in the four collaboration stages by the four different team types. During team knowledge construction the face-to-face heterogeneous teams spent longer in this stage than the asynchronous, distributed heterogeneous teams. During collaborative team problem solving the asynchronous, distributed teams spent more time problem solving than the face-to-face teams. The faceto-face heterogeneous teams had a significantly higher frequency of utterances, $F(3,80)=$ $5.64772, p=0.025802$, regarding team knowledge construction than the other three team types. Figure 6 illustrates the two cognitive states that were significantly different during team knowledge construction between the face-to-face and asynchronous, distributed teams. The asynchronous, distributed teams spent significantly more time understanding the problem compared to face-to-face teams, while the face-to-face teams spent more time building team knowledge compared to asynchronous, distributed teams. Figure 7 illustrates that during team problem solving, face-to-face teams spent significantly more time using conventions to transfer meaning than asynchronous, distributed teams while the asynchronous, distributed teams spent more time developing solution alternatives than face-to-face teams. There was no significant difference between the four-team types 


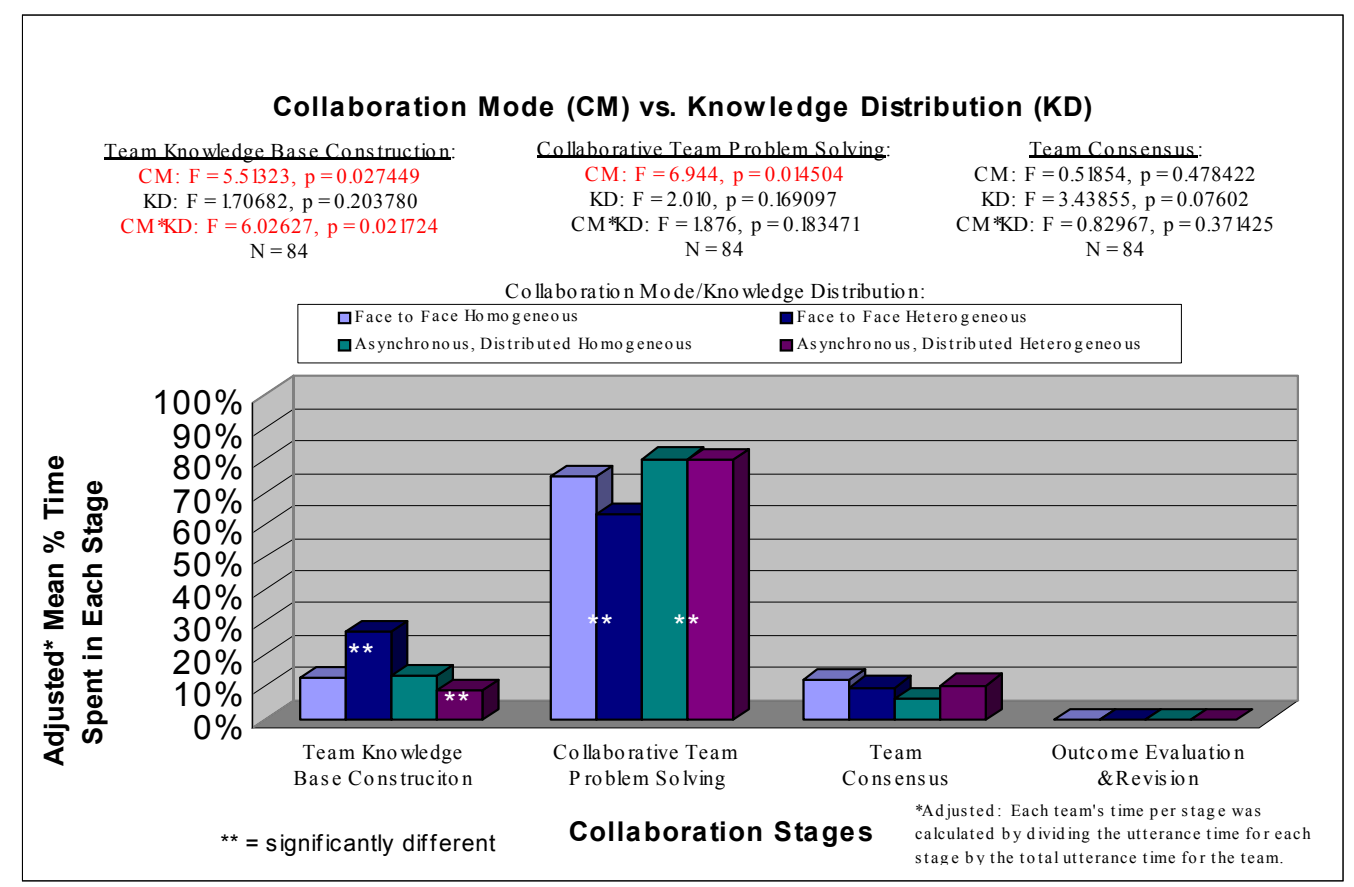

Figure 5: Mean Percent Time by Collaboration Stage.

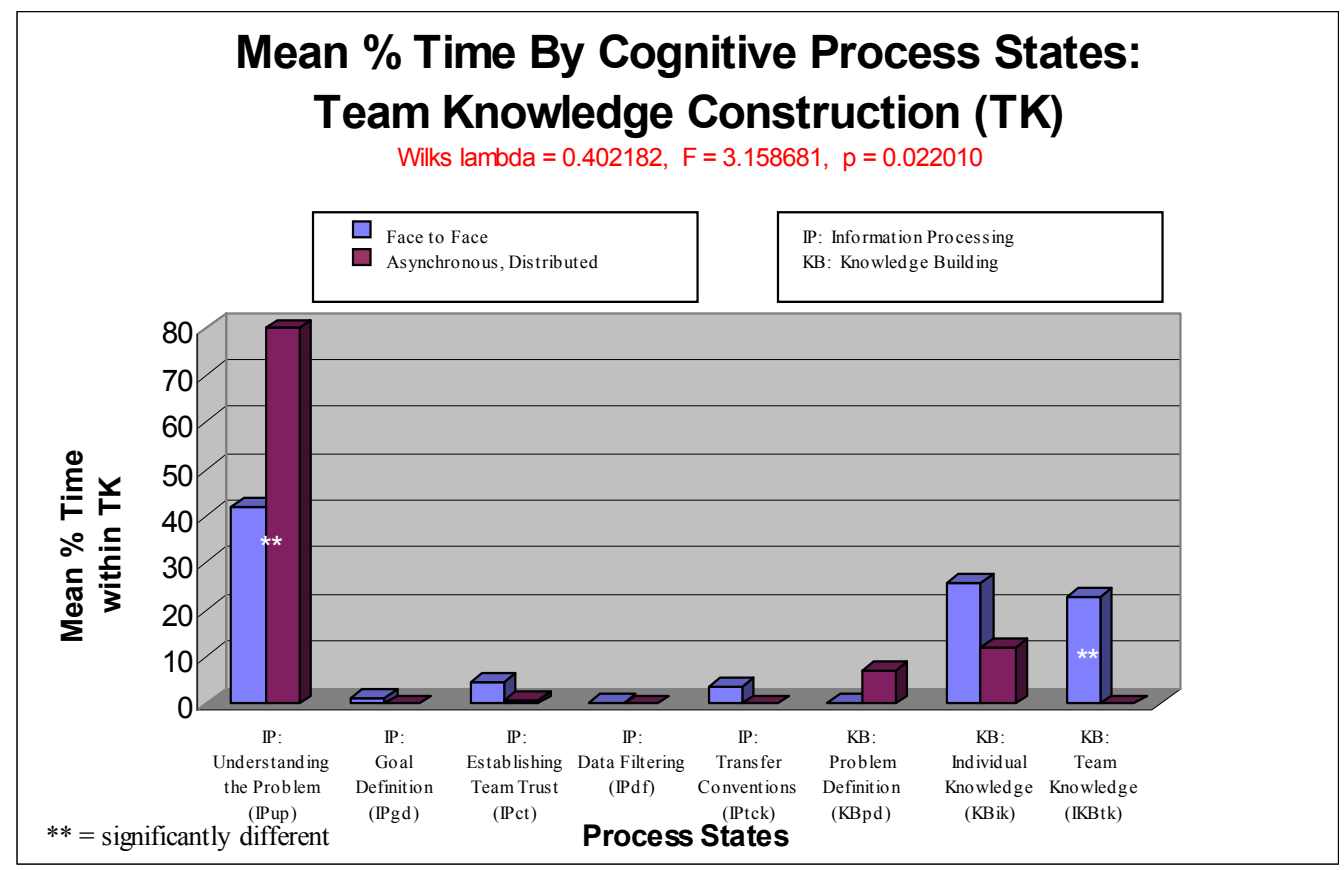

Figure 6: Mean Percent Time in Team Knowledge Construction. 


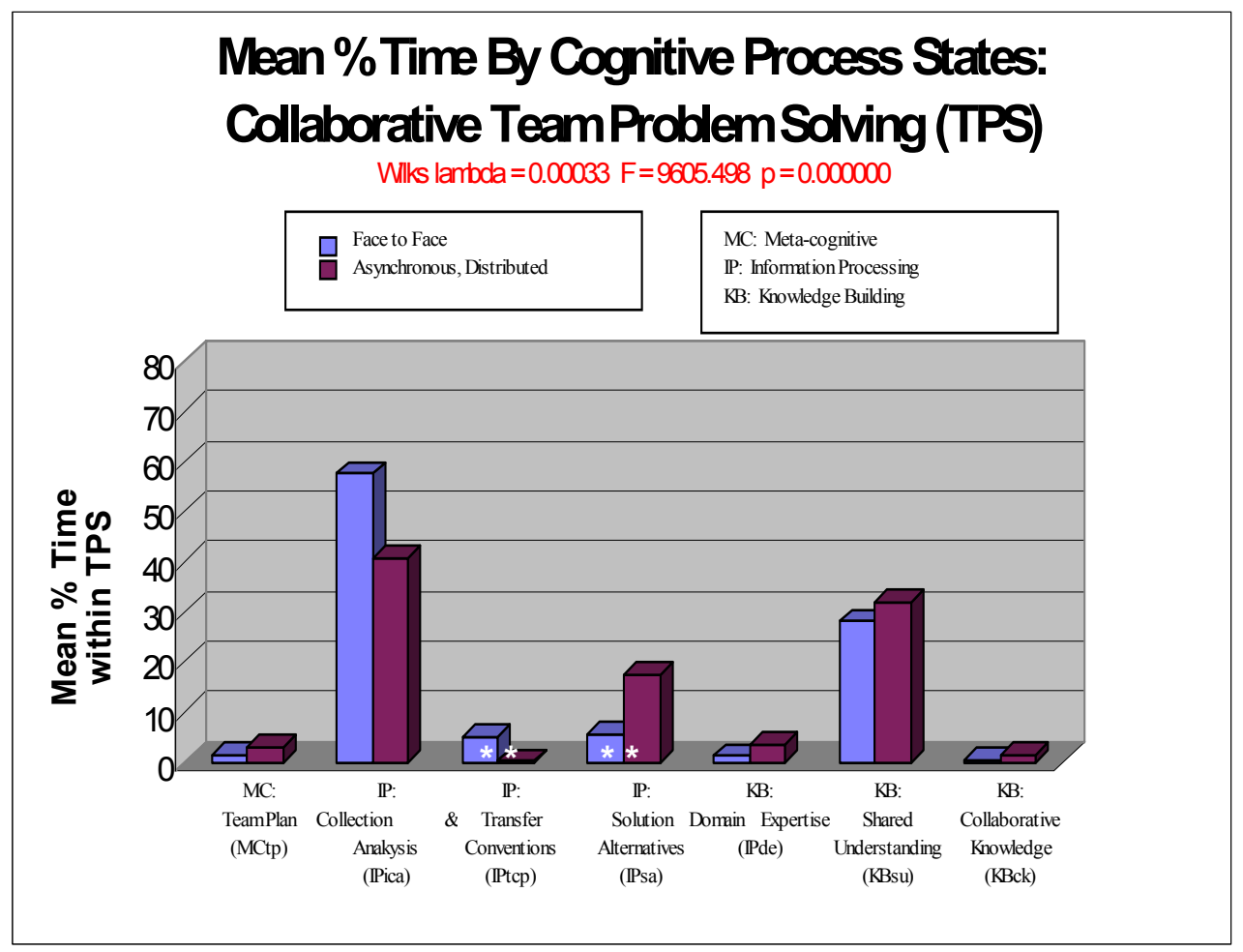

$* *$ = significantly different

Figure 7: Mean Percent Time in Collaborative Team Problem Solving.

in the cognitive processes used during team consensus, Wilks lambda $=0.815855, \mathrm{~F}=$ 1.65519, $\mathrm{p}=0.205648$. The transition probabilities for the collaboration stages and cognitive processes are presented in Figures 8 and 9 respectively.

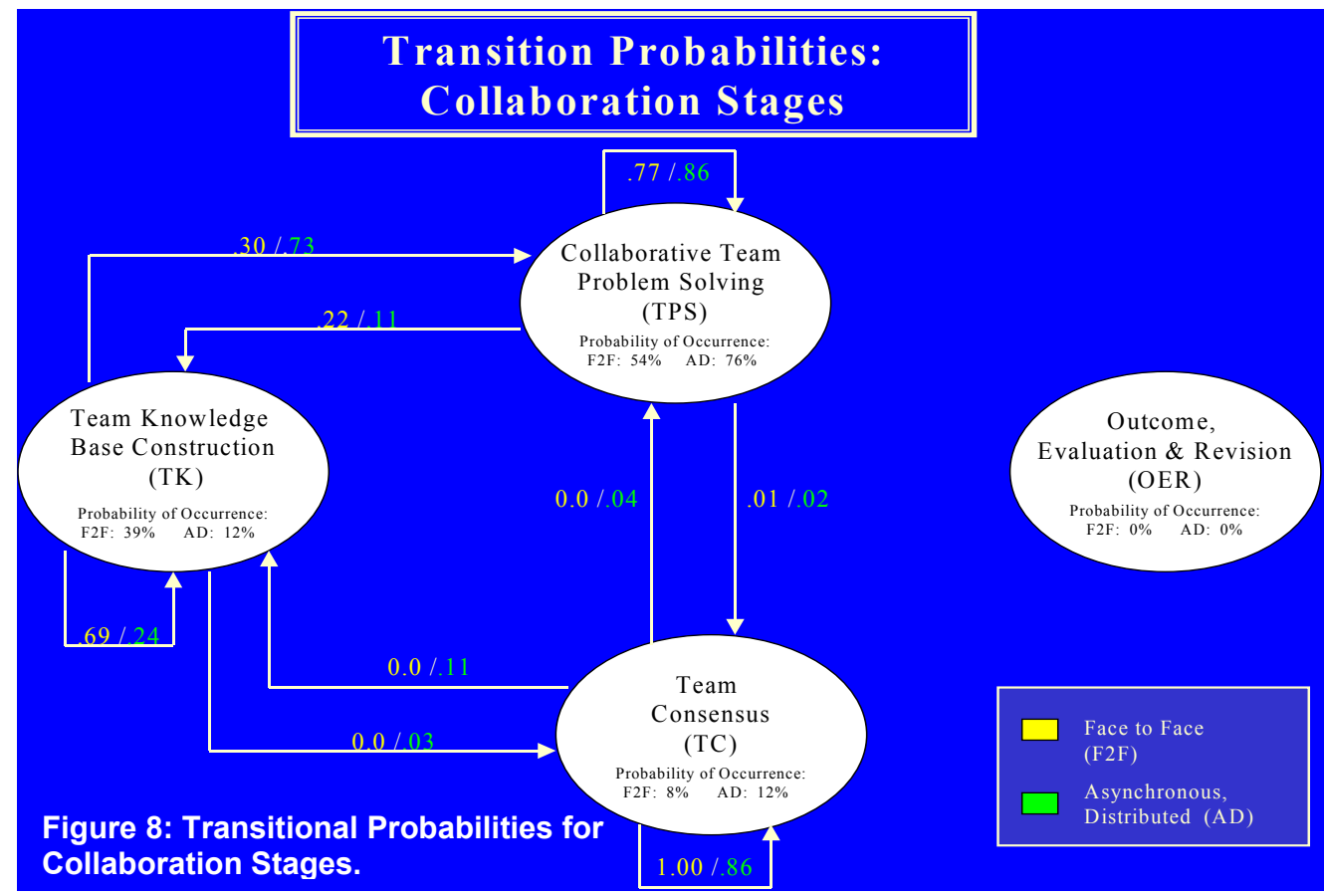




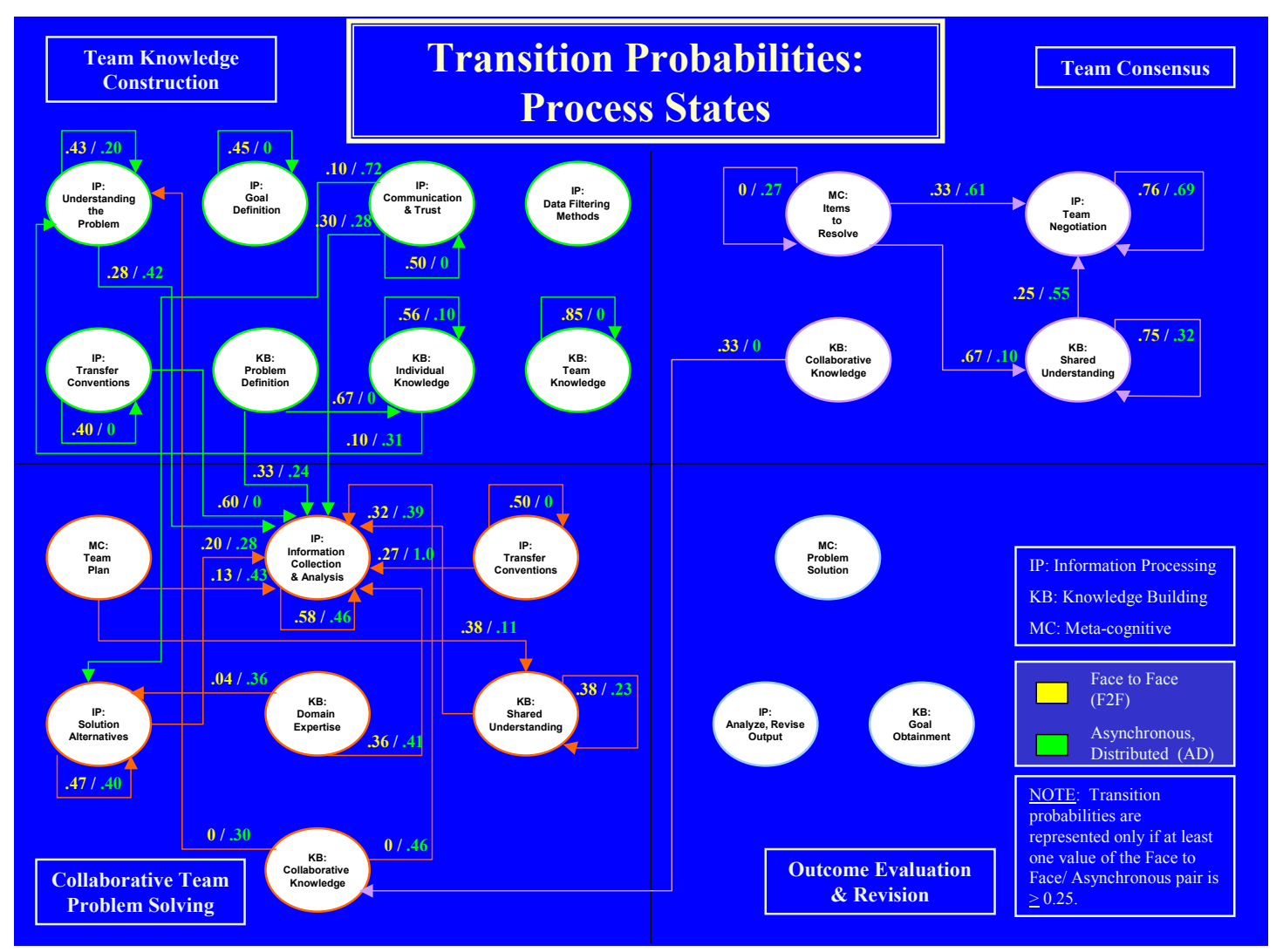

Figure 9: Transition Probabilities for Cognitive Processes

\section{Discussion}

Both face-to-face and asynchronous, distributed teams demonstrated behavior that supports the existence of Team Knowledge Construction, Collaborative Problem Solving and Team Consensus stages during collaborative problem solving. The empirical data showed that the stages are task dependent as the Outcome Evaluation \& Revision stage was not used. The significant cognitive process states were Understanding the Problem, Individual Knowledge and Team Knowledge development, Conventions for Transferring Meaning, and developing Solution Alternatives. Knowledge Distribution (homogeneous / heterogeneous information) significantly influenced how team knowledge was developed. Asynchronous, distributed teams spent more time in Collaborative Problem Solving stage than face-to-face teams. It appears to be more difficult to solve collaborative problems with these types of teams even though collaboration environment is inherently more structured. Need to determine why these teams spend more time so collaborative problem solving can be facilitated. Face-to-face teams used conventions to transfer meaning (e.g. yellow stickers, maps) whereas asynchronous, distributed teams did not use conventions. Need ways to easily create conventions with asynchronous, distributed teams (another area for agent support). Face-to-face teams demonstrated mostly a linear path between team knowledge construction, team problem solving and team consensus whereas asynchronous, distributed teams showed a non-linear path with feedback loops (area for agent support). In summary, the empirical data from this study 
has shown major differences between face-to-face and asynchronous, distributed teams in both the stages and the cognitive processes used during team collaboration. Based on these differences, capabilities need to be incorporated within new collaboration tools that facilitate asynchronous, distributed team collaboration.

\section{Acknowledgements}

Funds for the support of this research were provided by the Office of Naval Research, Code 342, Dr. Michael Letsky.

\section{References}

Anderson, J.R. (1993). Rules of the mind. Hillsdale, NJ: Lawrence Erlbaum Asociates.

Chomsky, N. (1957). Syntactic structures. The Hague: Mouton.

Churchland, P.M. (1989). A neurocomputational perspective: The nature of mind and the structure of science. Cambridge, MA: Bradford/MIT Press.

Cooke, N.J. (2003). Measuring collaborative cognition. Collaboration and knowledge management workshop proceedings, January 14-16, 2003. Office of Naval Research, Human System Department, Arlington, VA.

Cowen, M. (2003). Noncombatant evacuation operation (NEO) scenario. Unpublished manuscript. (available from Space and Warfare System Center, Code 244210, 53345 Engineer Street, San Diego, CA)

Cristian, F. (1996). Synchronous and Asynchronous Group Communication. Communications of the ACM, 39 (4). P. 88 - 97.

Sternberg, R. J., \& J.E. Davidson (Eds.) (1986). Conceptions of giftedness. New York: Cambridge University Press.

Davidson, J.E. \& Sternberg, R.J. (1984). The role of insight in intellectual giftedness. Child Quarterly, 28, 58-64.

Davidson, J.E., Deuser, R., \& Sternberg, R.J. (1994). The role of metacognition in problem solving. In J. Metcalfe \& A.P. Shimamura (Eds.), Metacognition.Cambridge, the MIT Press.

Descartes', R. (1641). Descartes' meditations. (D. Manley \& C. Taylor, Ed. and trans.). Retrieved June 2, 2003. (http://philos.wright.edu/descartes/meditations.html)

Feigenbaum, E. A., \& Feldman, J. (1963). (Eds.) Computers and Thought. New York: McGraw- Hill.

Goodman, B., Geier, M., Haverty, L., Linton, F., \& McCready, R. (2001). A Framework for Asynchronous Collaborative Learning and Problem Solving. In J. D. Moore et al. (Eds.), Artificial Intelligence in Education. IOS Press.

Greeno, J.G., \& Simon, H.A. (1988). Problem solving and reasoning. In R.C. Atkinson, R.J. Herrnstein, G. Lindzey, and R.D. Luce (Eds.), Stevens' handbook of experimental psychology (2nd ed., Vol. II, pp. 589-672). New York, NY: Wiley.

Flower, L.S. and Hayes, J.R. (1981). A cognitive process theory of writing. College composition and communication, 32(4), pp.365-387.

Hurley, John (2002). Towards a Cognitive Organizational Framework for Knowledge Management an ONR/SPAWAR Systems Center San Diego Workshop, Cognitive Elements of Effective Collaboration. San Diego, CA.

Information Management Strategic Plan (1999). Version 2, Department of Defense, Chief Information Officer. 
Jensen, J. A. (2002) Joint Tactics, Techniques, and Procedures for Virtual Teams. Assistant Deputy for Crisis Operations, USCINCPAC (J30-OPT), Camp H. M. Smith Joint Vision 2010, Chairman of the Joint Chiefs of Staff, Pentagon, Washington, DC, 2002.

Kant, I. (1781). The Critique of Pure Reason.(trans. Norman Kemp Smith, New York: St. Martin's Press, 1965).

McNeese, M. D., Rentsch, J. R., \& Perusich, K. (2000). Modeling, Measuring and Mediating Teamwork: The Use of Fuzzy Cognitive Maps and Team Member Schema Similarity to Enhance BMC3I Decision Making. IEEE International Conference on Systems, Man and Cybernetics, p 1081 - 1086. NY: Institute of Electrical and Electronic Engineers.

Minsky, M. (1981). A framework for representing knowledge. In J. Haugeland (Ed.), Mind design. Cambridge, MA: MIT Press.

Newell, A. \& Simon, H.A. (1972). Human problem solving. Englewood Cliffs, NJ: Prentice Hall.

Noble, David (2002). Cognitive-Based Guidelines for Effective Use of Collaboration Tools. Cognitive Elements of Effective Collaboration. University of San Diego, 15-17. Coalition Planning Guide (2003). (available from M. Cowen, Space and Warfare Systems Center, Code 244210, 53345 Engineer Street, San Diego, CA).

Orasanu, J. \& Salas E.(1992) Team Decision Making in Complex Environments. In Klein, G., Orasanu, J., \& Calderwood, R. (eds.). Decision Making in Action: Models and Methods. Norwood, NJ: Ablex Publishing Corp.

Pea, R. D \& Hawkins, J., (1987). Tools for bridging everyday and scientific thinking. Journal for Research in Science Teaching, 24(4), 291-307.

Piaget, J (1975) Piaget's Developmental Psychology. In Hilgard, E. \& Bower, G. (Ed.) Theories of Learning. The Century Psychology Series, Prentice-Hall, Inc. Englewood Cliffs, N.J.

Rogers, Y. \& Ellis, J. (1994). Distributed Cognition: an alternative framework for analyzing and explaining collaborative working. Journal of Information Technology, 9 (2), 119-128.

Rosenberg, A. 1988. Philosophy of Social Science. Westview.

Rumelhart, D.E. (1990). Brain style computation: Learning and generalization. In S.F. Zornetzer, J.L. Davis, and C. Lau (Eds.), An introduction to neural and electronic networks. San Diego: Academic Press.

Shannon, C. E. (1949). A mathematical theory of communication. Bell System Technical Journal, vol. 27, pp. 379-423 and 623-656.

Skinner, B.F. (1989). The origins of cognitive thought. American Psychologist, 44, 13-18.

Skinner, B.F. (1985). Cognitive science and behaviourism. British Journal of Psychology, 76, 291-301.

Stahl, Gerry (2000). A Model of Collaborative Knowledge-Building. In B. Fishman \& S. O'Connor-Divelbiss (Eds.), Fourth International Conference of the Learning Sciences. Mahwah, NJ: Erlbaum, 70-77.

Stasser, G., Stewart, D. D., \& Wittenbaum, G. M. (1995). Expert roles and information exchange during discussion: The importance of knowing who knows what. Journal of Experimental Social Psychology, 31, 244-265. 
Turing, A.M. (1936) On Computable Numbers, with an Application to the Entscheidungsproblem. Proceedings of the London Mathematical Society, second series, vol. 42, part 3, 230-265.

Warner, N.W., Letsky, M. and Cowen, M. (In Press). Structural model of team collaboration.

Warner, N.W., Vanderwalker, S. and Verma, N. (2002). State of the art review of humanhuman collaboration research: an integrated, multidisciplinary perspective. In collaboration and knowledge management workshop proceedings, January 14-16, 2003. Office of Naval Research, Human System Department, Arlington, VA.

Weiner, Norbert (1948), Cybernetics, John Wiley and Sons, Inc., New York.

Wickens, C.D. (1992). Engineering Psychology and Human Performance (2nd Ed.). Harper Collins: New York 
Appendix A: Coding Categories and Definitions for Collaboration Stages and Cognitive Processes

\section{Collaboration Stages Categories \& Definitions}

- Team Knowledge Base Construction (TK): "Team members reading, clarifying information and understanding the problem."

* Defining the problem (realizing what they have to accomplish ---

"We need to come up with a solution in 30 minutes.")

* Clarifying the facts --- "What did they say about Joe's hearing?

- Collaborative Team Problem Solving (TPS): "Team members working together to develop solution options to the problem."

* Analyzing the facts to come up with a solution - - - "I think Joe did it because he tried to blame everything on Tim."

- Team Consensus (TC): "Team negotiation of solution option and final agreement by all team members on a particular option."

* Agreeing on the final solution - - " "Do we all agree that Joe did it?"

- Outcome Evaluation \& Revision (OER): "Team evaluation of selected solution option against problem solving goal. Team revises solution option if option does not meet goal."

* Choosing to accept the final decision or revise it - - - "I know we all agreed on Tim, but I think we better take a closer look at Joe."

- Additional Stages (MISC): "Other unique team behavior not described in the above categories."

* Need to describe unique behavior and label stage.

\section{Cognitive Process States: Categories \& Definitions}

Team Knowledge Base Construction: (TK) "Team members reading clarifying and understanding the problem to be solved."

IPup: Information Processing (understanding problem) = stating the facts without applying that knowledge to any possible solution.

"Tim's muffler was loud"

IPgd: Information Processing (goal definition) = defining the team goal.

"Our goal is to determine who killed Mr. Gill"

IPct: Information Processing (communication \& trust) = establishing team trust.

"I understand why you thought it was Tim."

IPdfm: Information Processing (data filtering methods) = team uses methods to sort data.

"using yellow note pads to sort data into categories" 
IPtck: Information Processing (transfer conventions) $=$ team uses methods to transfer meaning to other team members during knowledge construction.

"team uses map of Mr. Gill's house to understand distances between objects.

KBpd: Knowledge Building (problem definition) = defining the problem (realizing what they have to accomplish)

"We need to come up with a solution in 30 minutes"

KBik: Knowledge Building (indiv. knowledge) = individual clarifying the facts; asking for clarification.

"What did they say about Joe's hearing"

KBtk: Knowledge Building (team knowledge) = team clarifying facts

"Billy left the coffee shop at 7:00am"

"No that was Eddie that left the coffee shop at 7:00am

"Yes that was Eddie"

\section{Collaborative Team Problem Solving: (TPS) "Team members working together to} develop solution options to the problem."

MCtp: Meta-cognitive (team plan) = establishing a plan of approach.

"ADHES 2, why don't you say who you think it is, then I'll say who I think it is." IPica: Information Processing (information collection $\&$ analysis) $=$ collecting and analyzing the facts to come up with a solution but no specific solution mentioned.

"The killer seems to blame everything on Tim."

IPtcp: Information Processing (transfer conventions) = team uses methods to transfer meaning to other team members during team problem solving.

"Using maps or yellow stickers to transfer meaning to team members"

IPsa: Information Processing (solution alternatives) = developing, rationalizing and discussing solution alternatives.

"I think Eddie did it?"

KBde: Knowledge Building (domain expertise) = team members state their own domain knowledge and apply that knowledge to the problem.

"Yes I am a Maryland State Trooper and they is no concrete evidence for Billy killing Mr. Gill."

KBsu: Knowledge Building (shared understanding) = using facts to justify a solution.

"I think Eddie did it because he was hard of hearing and had his fingerprints on the crowbar."

KBck: Knowledge Building (collaborative knowledge) $=$ Convincing others of a specific thought without absolute consensus of the final solution.

"You're right. I didn't think about that."

\section{Team Consensus: (TC) "Team negotiation of solution option and final agreement} by all team members on a particular option."

MCitr: Meta-cognitive (items to resolve) = team stating remaining items to resolve.

"We need to compare Mickey's and Eddie's location times with Billy's"

IPtn: Information Processing (team negotiation) = team negotiation of solution alternatives ending in a final solution.

"I believe it is Eddie because he was hard of hearing"

"I concur which also means Eddie was lying about where is was" 
"I also think it was Eddie because of his fingerprints on the crowbar.

"We all in consensus, it was Eddie"

KBckc: Knowledge Building (collaborative knowledge) = convincing others of a specific thought without absolute consensus of the final solution.

"You're right. I didn't think about that."

KBsuc: Knowledge Building (shared understanding) = using facts to justify a solution.

"It couldn't have been Tim because his muffler was loud and the car at the coffee shop had a quiet muffler."

\section{Outcome Evaluation \& Revision: (OER) "Team evaluation of selected solution} option against problem solving goal. Team revises solution option if option does not meet goal."

MCps: Meta-cognitive (problem solution) = compare problem solution against goal(s).

"Are we done or do we want to talk about it some more?"

IPar: Information Processing (analyze, revise output) = analyze final solution option and revise if necessary.

"I know we all agreed on Tim, but I think we'd better take a closer look at Joe." KBgo: Knowledge Building (goal obtainment) = understand if solution option fits specific goal criteria.

" the murder had a truck with a loud muffler, was at Mr. Gills house around 10:30am, and pushed Mr. Gill down the porch steps". 


\section{Appendix B: Glossary of Terms}

Collaboration: the process of shared creation; two or more individuals with complementary skills interacting to create a shared understanding that none had previously possessed or could have come to on their own. The cognitive aspects of joint problem solving for the purpose of attaining knowledge sufficient to complete the common task.

Shared Understanding: The process of reaching team agreement about the characteristics and meaning of the situation at hand within the bounds of the objectives of the collaborative activity (decision making, intelligence analysis, COA selection, etc.). Not all team members will have equal depth and breadth in all supporting knowledge but each has sufficient knowledge to complete his role as a team member.

Asynchronous, Distributed Collaboration: a cohesive group of individuals working at different locations and at different times to solve a common task.

Mental Model: a knowledge structure that represents information.

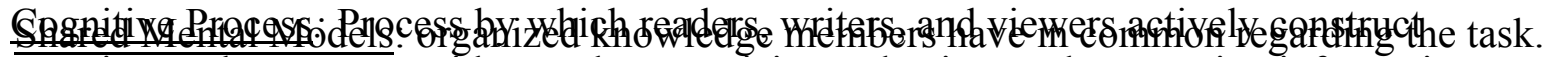
meaning as they engage with texts by organizing, selecting, and connecting information; making inferences; and performing acts of interpretation.

Computational Models: Calculational tool that implements a set of mathematical equations designed to represent a conceptual model.

\section{Conceptual Models:}

(1) Set of qualitative assumptions used to describe a system (or part thereof). These assumptions may cover the geometry and dimensionality of the system, initial and boundary conditions, time dependence, and the nature of the relevant physical, chemical and biological processes and phenomena.

(2) Consists of a set of assumptions that reduce the real problem and the real domain to simplified versions that are satisfactory in view of the modeling objectives and the associated problem.

Consensus: Opinion or position reached by the group as a whole.

Data: Factual information (as measurements or statistics) used as a basis for reasoning, discussion, or calculation. Data on its own has no meaning, but becomes information when it is interpreted.

Decision-Making: Form of problem solving in which one tries to make the best choice from among alternative judgments or courses of action.

Human-Agent Interface: A connection point that allows for the interaction between a user and software, which carries out some set of operations on behalf of a user or another 
program with some degree of independence or autonomy, and in so doing, employ some knowledge or representation of the user's goals or desires.

Knowledge Building:

(1) Process through which we increase both our individual and our common understanding.

(2) Theory of learning, which emphasizes the collaborative construction of knowledge by a group of learners.

Situational Awareness: Person's mental model of the current state of a dynamic environment; the perception of the elements in the environment within a volume of time and space, the comprehension of their meaning and the projection of their status in the near future.

Transactive Memory: Consists of the collection of individual understandings and the team mechanisms to exchange information, which update these individual understandings. 